Antenna pattern of DUAL detectors of gravitational waves and its exploitation in a network of advanced interferometers

This article has been downloaded from IOPscience. Please scroll down to see the full text article.

2008 Class. Quantum Grav. 25195018

(http://iopscience.iop.org/0264-9381/25/19/195018)

View the table of contents for this issue, or go to the journal homepage for more

Download details:

IP Address: 194.94.224.254

The article was downloaded on 02/06/2010 at 10:07

Please note that terms and conditions apply. 


\title{
Antenna pattern of DUAL detectors of gravitational waves and its exploitation in a network of advanced interferometers
}

\author{
Paola Leaci ${ }^{1,4}$, Tito Dal Canton ${ }^{2,3}$, Antonello Ortolan ${ }^{2}$ and \\ Giovanni A Prodi ${ }^{1}$ \\ ${ }^{1}$ Department of Physics, University of Trento and INFN Trento, I-38100 Povo (Trento), Italy \\ 2 Department of Physics, University of Padova, I-35100 Padova, Italy \\ ${ }^{3}$ INFN Laboratori Nazionali di Legnaro, I-35020 Legnaro (Padova), Italy \\ E-mail: leaci@science.unitn.it and paola.leaci@aei.mpg.de
}

Received 12 May 2008, in final form 21 July 2008

Published 16 September 2008

Online at stacks.iop.org/CQG/25/195018

\begin{abstract}
We investigate the directional sensitivity to plane gravitational waves (GWs) of DUAL detectors of cylindrical shape. Calculations make use of the finite element method to simulate the responses to the GW Riemann tensor of a singlemass DUAL (SMD) and of a tapered cylinder (TC) in their wide sensitivity bandwidth. We show that one SMD or a pair of TCs is able to cover both GW polarization amplitudes from almost all incoming directions. We discuss the achievable enhancement in tackling the inverse problem for high frequency $[\sim(2-5) \mathrm{kHz}] \mathrm{GWs}$ by adding a TC detector to the future advanced LIGOVIRGO network.
\end{abstract}

PACS numbers: $04.80 . \mathrm{Nn}, 95.55 . \mathrm{Ym}$

(Some figures in this article are in colour only in the electronic version)

\section{Introduction}

The search for gravitational waves (GWs) represents a major challenge in experimental physics. A crucial problem of the GW detection is the reconstruction of the wave parameters (direction and polarizations) from the detector outputs, i.e. solving the inverse problem. For GW bursts this is even more problematic due to the lack of knowledge of their time-frequency contents and to the very high rates of spurious signals in ground-based detectors. The observational results from burst searches have been either autonomous [1-3] or triggered by other astrophysical observations [4-6]. A single detector readout cannot measure both polarization components of a GW, so to assign a GW signal we must consider the synthesis of several detector outputs

\footnotetext{
4 Author to whom all correspondence should be addressed. 
[7]. Only a spherical GW detector [8,9] could in principle solve the inverse problem by itself. The inverse problem has already been discussed for networks of GW detectors: three $[7,10]$, four and five detectors, either resonant or interferometric [11], giving rise to novel data analysis methods (e.g. based on aperture synthesis [12], constrained likelihood [13] or null streams [14]). The network data analysis of GW detectors requires the knowledge of the directional (angular) response of the detectors, the so-called antenna pattern.

In general, it is not easy to accurately reconstruct a GW signal using the noisy data of a detector network as its amplitude is expected to be of the same order of the noise fluctuations [14].

The current most sensitive searches for GWs are carried out by the LIGO-VIRGO laser interferometers, which have recently achieved their design sensitivity goal [15-17], exhibiting their best sensitivity in the range $\sim(100-1000) \mathrm{Hz}$.

Due to the actual detector positions and orientations, the existing network of interferometers is almost blind to one of the two GW polarization amplitudes and the inverse problem is ill-conditioned [18]. The main motivation of this paper is the fact that the integration of one single-mass DUAL (SMD) [19] or one tapered cylinder (TC) [20] can considerably improve the network performances with respect to sky coverage and spurious rejection, at least in frequency bands where detector sensitivities can be of the same order of magnitude.

In this paper, we compute the response functions to GWs of SMD and TCs, equipped with geometrically selective readouts [21,22]. It has been shown [19] that a SMD, consisting of a hollow cylinder optimized for the frequency range $\sim(2-5) \mathrm{kHz}$, can offer all the advantages of a previous configuration, namely a DUAL detector [22], but with a simpler practical realization. To make such a SMD configuration feasible, noise matching between the test mass and the readout must be achieved, for instance, by using realistic capacitive readouts mounted on a hollow cylinder modified with transverse displacement concentrators (whips) [20]. The resulting TC detector exhibits a sensitivity comparable to that of an ordinary SMD detector [20], with a standard quantum limit sensitivity comparable to the design sensitivity of advanced interferometers in the $\sim(2-5) \mathrm{kHz}$ band [15].

This paper is organized as follows. In section 2, we derive the expression for the GW equivalent force, coming from any direction of the sky and with arbitrary polarization and describe how the antenna pattern is computed by the finite element method (FEM). In section 3, we present the case study of the SMD and TC detectors. Section 4 describes the antenna patterns of the SMD and TC detectors and comments the selectivity of their response to a GW. In section 5, we discuss the GW inverse problem, by providing a brief overview of its applications to a detector network. Section 6 presents the significant improvement of the network performances that one can get by adding a TC detector to the advanced LIGO-VIRGO interferometers. Finally, conclusions are drawn in section 7.

Throughout this paper, the bold fonts represent matrices and vectors, the middle-dots indicate the ordinary matrix product and the superscript $(\cdot)^{T}$ represents the transpose of a matrix. Moreover, we use the Einstein convention, i.e. repeated indices are implicitly summed over. We assume GW wavelengths to be much larger than the cylindrical detectors' size [23].

\section{Interaction of GWs with a detector}

Let us assume that the gravitational radiation impinges on a $\mathrm{GW}$ detector of cylindrical shape, coming from a direction $k$ and with an arbitrary polarization. To calculate the detector angular sensitivity, we choose a coordinate system $\left\{x_{1}, x_{2}, x_{3}\right\}$ with the $x_{3}$-axis (i.e. the local vertical) aligned along the cylinder symmetry axis and origin in the center of mass of the cylinder. 
Given the short interaction time with the GW and the small volume of the detector, the detector reference frame can be considered a reasonable approximation of the Fermi normal coordinates system [23]. According to the geodesic deviation equation, a test particle of mass $M$, position vector $\boldsymbol{x}$ (i.e. at a distance $\|\boldsymbol{x}\|$ from the origin) experiences a force given by

$$
f_{i}=M c^{2} R_{i j} x_{j}
$$

where $R_{i j} \equiv R_{0 i 0 j}$ is the tidal matrix and $R_{0 i 0 j}$ are components of the Riemann curvature tensor on the detector reference frame [23]. Assuming the linearized theory of general relativity on the Minkowski spacetime, the Riemann tensor describing a plane GW reads

$$
R_{i j}=-\frac{1}{2 c^{2}}\left[\ddot{h}_{+}(t) e_{i j}^{+}+\ddot{h}_{\times}(t) e_{i j}^{\times}\right]
$$

where $h_{+}(t)$ and $h_{\times}(t)$ are the independent amplitudes of the wave and $e_{i j}^{+, \times}$are the polarization tensors. They have simple components in the wave basis:

$$
e^{+}=\left[\begin{array}{ccc}
1 & 0 & 0 \\
0 & -1 & 0 \\
0 & 0 & 0
\end{array}\right] \quad e^{\times}=\left[\begin{array}{ccc}
0 & 1 & 0 \\
1 & 0 & 0 \\
0 & 0 & 0
\end{array}\right] .
$$

Their components in a detector-fixed basis depend on the direction of the incoming wave $\boldsymbol{k}=(\cos \varphi \sin \theta, \sin \varphi \sin \theta, \cos \theta)$, with $\theta$ and $\varphi$ being spherical angles in $\left\{x_{1}, x_{2}, x_{3}\right\}$

In order to evaluate the directional sensitivity of the SMD/TC elastic cylinders, we performed, by means of the ANSYS code [24], three-dimensional FEM full method ${ }^{5}$ harmonic analyses.

The SMD is sketched in figure 1, while its readout configuration is shown in figure 2. It is worth noting that the combination of $\xi_{1}^{\mathrm{SMD}}$ with a second readout $\xi_{2}^{\mathrm{SMD}}$, rotated with respect to the first by $\pi / 4$ and shown in figure $2\left(a_{2}\right)$, allows one to measure both $\mathrm{GW}$ polarization states. The FEM computes the average deformation of the detector along four stripes in the inner hole surface. These deformations are then combined as described in [22]: the expansion along one diameter is summed to the shrinking along the perpendicular diameter (this is very similar to what accomplished in a Michelson interferometric detector with arms at $90^{\circ}$ ). In this way, the resulting detector response, $\xi(\omega)$, is sensitive only to quadrupolar deformations, rejecting other deformation symmetries.

The resulting GW transfer function $H^{+, \times}(\theta, \varphi ; \omega)$ depends on the GW direction and frequency. In principle, it can be obtained in the frequency domain by a sum of the contributions of each normal mode of the detector weighted by the Lorentzian factor $\omega^{2} /\left[\left(\omega_{m}^{2}-\omega^{2}\right)+\mathrm{i} \omega_{m}^{2} \phi_{m}(\omega)\right][19,25]$, where $\omega_{m}$ represents the resonance angular frequency of the normal mode $m$. For frequency-independent losses, the phase lag $\phi_{m}$ is equal to the inverse of the detector mechanical quality factor $Q$ [26]. Since each normal mode of a resonant detector has its own directional sensitivity, generally it is not possible to separate the frequency and directional dependences of the GW transfer function in a wide sensitivity bandwidth. A different factorization of $H^{+, \times}$in general holds around different resonant frequencies. In the case of cylindrical DUAL detectors, however, the geometrical selectivity of the readout for quadrupolar modes allows one to factorize $H^{+, \times}(\theta, \varphi ; \omega)$ as $H^{+, \times}(\theta, \varphi ; \omega) \equiv F^{+, \times}(\theta, \varphi) G(\omega)$ over the very wide bandwidth of sensitivity $[19,20]$; here $G(\omega)$ and $F^{+, \times}$are the usual

5 The full method is the most common and accurate method used to determine the response of a structure to harmonically time-varying excitation. The FEM method is employed in solving approximately problems which involve partial derivatives. The main feature of the FEM method is the discretization (mesh) of the continuous body by using finite elements of simple shape [24]. A suitable mesh reduces the continuous system dynamics to algebraic equations that allow the calculation of the elastic body dynamical impedance. The ANSYS solver computes also the transfer functions from the input GW equivalent force to the output displacement readouts. 


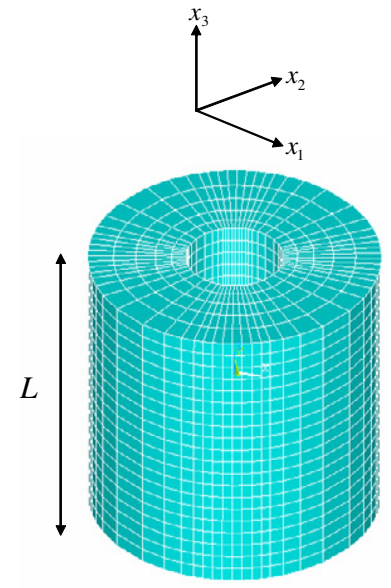

(a)

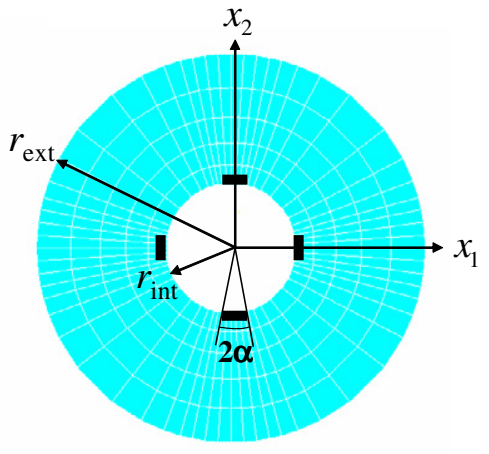

(b)

Figure 1. FEM-simulated SMD detector and readout. (a) The deformations due to a GW are measured along surface stripes in the inner hole extending over the entire cylinder height $L$ with an angular aperture $2 \alpha$. We used the value $\alpha=0.2 \mathrm{rad}$ as in [19]. (b) Transverse section of the detector where we have indicated with a black tile one of the sensing surfaces; $r_{\text {ext }}$ and $r_{\text {int }}$ are the outer and inner radii of the hollow cylinder.

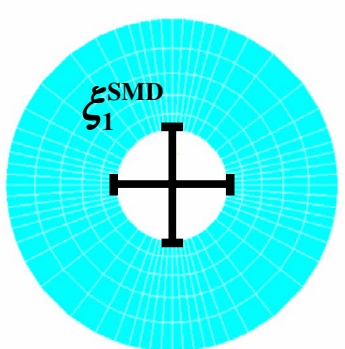

$\left(a_{1}\right)$

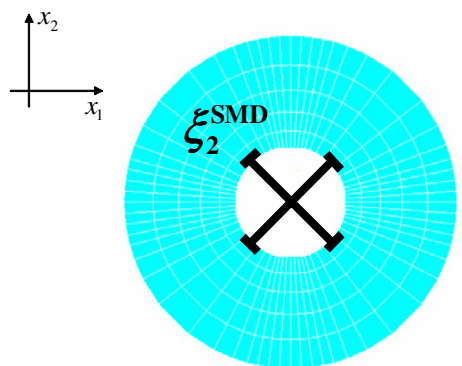

$\left(a_{2}\right)$

Figure 2. Detector transverse sections and readout measurement strategy. The measuring surfaces are constituted by independent readout configurations. The configurations in the panels $\left(a_{1}\right),\left(a_{2}\right)$ are sensitive to the quadrupolar modes of the test mass ( $\alpha=0.2 \mathrm{rad}$ was used). The joint use of such configurations allows one to detect both GW polarizations.

detector transfer function and antenna pattern, respectively. By definition, the antenna pattern $F^{+, \times}(\theta, \varphi)$ of a $\mathrm{GW}$ detector gives the dependence of the response on the wave direction and polarization.

The FEM analysis allows us to compute $H^{+, \times}(\theta, \varphi ; \omega)$ in the presence of complicated shapes of the elastic body or arbitrary readout surfaces with a different approach, i.e. without performing a normal mode expansion, but by computing directly the resulting deformation $\xi(\omega)$ with the external force field.

To handle non-resonant readout configurations, e.g. the evaluation of SMD antenna pattern, we must resort to this approach because it is more direct and accurate than considering the superposition of a very large number of normal modes, at the cost of a reasonable increase in computational requirements. 


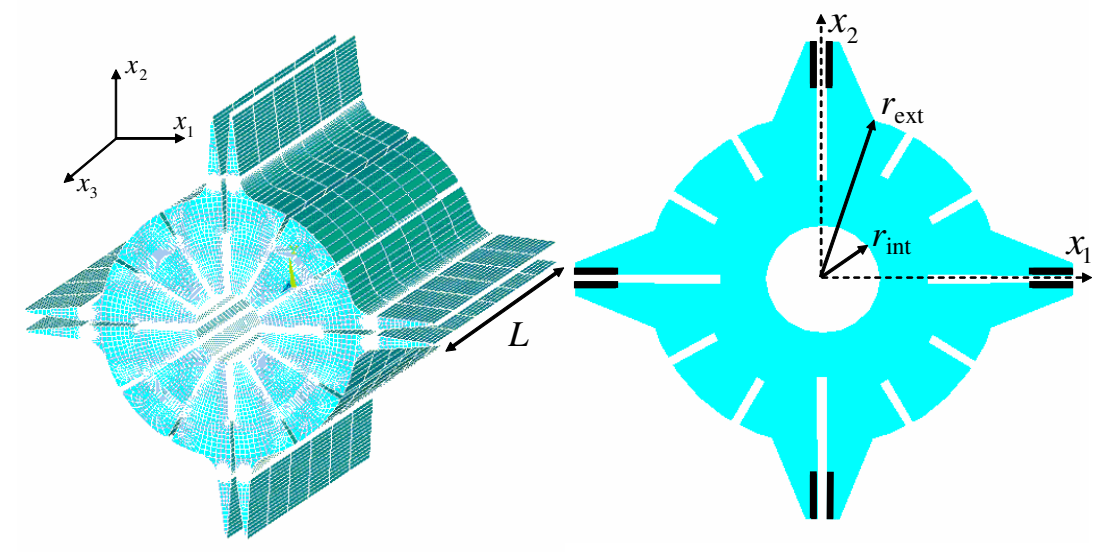

(a)

(b)

Figure 3. Geometrical parameters used for the FEM simulations of the Mo TC detector: $r_{\mathrm{ext}}=$ $0.35 \mathrm{~m}, r_{\text {int }}=0.091 \mathrm{~m}, L=1.19 \mathrm{~m} ; r_{\text {ext }}$ does not include the displacement concentrators. In particular, the whip length extends from the $r_{\text {ext }}$ up to $\sim 16 \%$ (the drawings are not in scale). The cylinder symmetry axis is aligned with the $x_{3}$-axis (i.e. the local vertical) and the whips are optimized to ensure a high mechanical gain. The sensing surfaces are indicated by the black segments at the end of each whip and make four gaps. Opposite gaps make a pair and related gap changes are summed together. The detector response is then the differential change in the two orthogonal pairs of gaps.

Table 1. Parameters used for the FEM simulations of the Mo cylinder in figure 1. We indicate the cylinder's outer and inner radii, and its length with $r_{\text {ext }}, r_{\text {int }}$ and $L$, respectively. The density, the Poisson's ratio and the quality factor of the Mo material are denoted by $\rho, \sigma$ and $Q$ [27], respectively.

\begin{tabular}{llllll}
\hline$r_{\text {ext }}(\mathrm{m})$ & $r_{\text {int }}(\mathrm{m})$ & $L(\mathrm{~m})$ & $\rho\left(\mathrm{kg} \mathrm{m}^{-3}\right)$ & $\sigma$ & $Q$ \\
\hline 0.5 & 0.15 & 3 & 10280 & 0.31 & $10^{7}$ \\
\hline
\end{tabular}

The FEM simulations are repeated for different GW directions $k$ sampled on a discrete grid of the angles $\theta$ and $\varphi$.

In our simulations, we assume elastic behaviour and take $h_{+, \times}$to be unity. This unphysical value is suitable to keep $\xi(\omega)$ large enough to avoid underflow problems due to the finite numerical accuracy of the FEM simulations.

\section{Case study on cylindrical DUAL GW detectors}

As a case study, we consider two types of three-dimensional models of cylindrical DUAL detectors made of molybdenum (Mo). Molybdenum is a material with low mechanical losses, useful to reduce the thermal noise contribution; in fact, the ratio between the temperature and quality factor is $T / Q \leqslant 5 \times 10^{-9} \mathrm{~K}$ for acoustic modes at $50 \mathrm{mK}$ [27]. The case study for the SMD (see figure 1) has been modelled with the parameters presented in table 1 . The model of the TC detector is described in figure 3.

In order to fully exploit the SMD sensitivity [19], both the transducer configurations of figures $2\left(a_{1}\right)$ and $\left(a_{2}\right)$ must be taken into account. Therefore, we consider such a cylinder equipped with the couple of these transducers, and we study their response to the two independent polarizations of the wave. Unfortunately, the noise stiffness required to optimize 


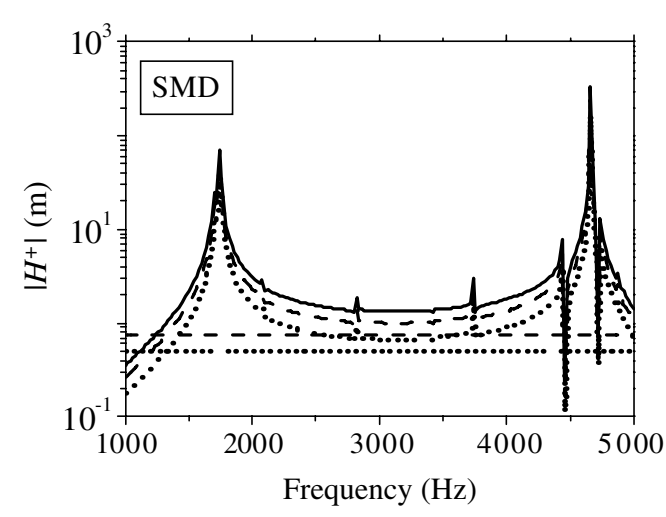

(a)

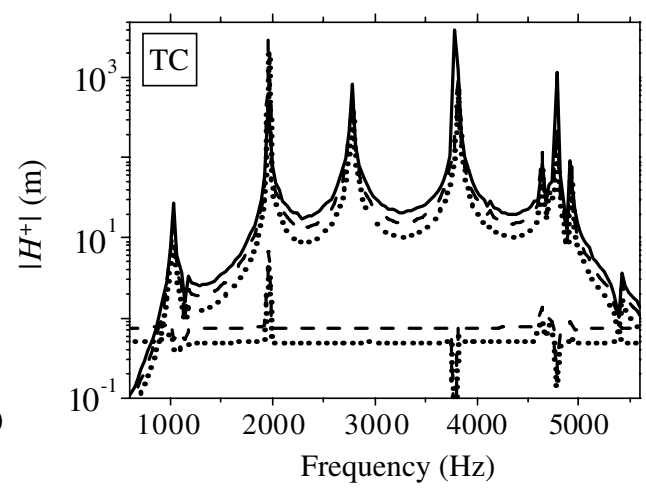

(b)

Figure 4. $\left|\mathrm{H}^{+}\right|$computed for the three-dimensional elastic models of SMD, equipped with a readout as in figure $2\left(a_{1}\right)$, and of TC (panels $(a)$ and $(b)$, respectively). These functions describe the dynamical response of the hollow cylindrical detector to a plus wave propagating in the direction: (i) $(\theta=0, \varphi=0)$, solid line; (ii) $\left(\theta=\frac{\pi}{4}, \varphi=0\right)$, dashed line; (iii) $\left(\theta=\frac{\pi}{2}, \varphi=0\right)$, dotted line. The horizontal dashed and dotted lines represent the ratio between the $\left|H^{+}\right|$of a wave travelling in the directions described in (ii) and (i), (iii) and (i), respectively. The frequency is defined by $v=\omega /(2 \pi)$. Similar plots also hold for the cross polarization.

the SMD sensitivity is two or three orders of magnitude larger than the noise stiffness of any realistic readout, both electromechanical or optomechanical.

On the TC detector only one transducer configuration can be provided. The more complex model geometry of the TC detector has been developed to fulfil a crucial experimental requirement: the noise matching between the test mass and the readout [20]. This matching is also necessary to fully exploit the readout sensitivity, up to its standard quantum limit [28]. As sketched in figure 3, displacement concentrators can be obtained by radial grooves and properly tapered whips on the external surface of the hollow cylinder. This new design allows us to achieve an amplification of the mechanical displacement on a large band by a factor of $\sim 20$ with respect to the standard SMD configuration [20]. Here the whips provide an amplification of their transverse displacement, making a tangential sensing of the test mass deformations possible. In fact, the readout measures the gaps between the tips of two-faced whips (black segment in figure 3(b)). These faced surfaces could represent either the plates of a capacitor [20, 29, 30] or the mirrors of a Fabry-Perot cavity [31-33].

We have shown in [20] that a TC equipped with the external readout exhibits the same directional sensitivity as a SMD equipped with only one readout (see figure $2\left(a_{1}\right)$ ), i.e. the same antenna pattern as an interferometric detector with two $90^{\circ}$ arms. Such TC is not omnidirectional, since it exploits only one single readout system, because of an unavoidable symmetry break with respect to rotation by a $\varphi=\pi / 4$ angle. To recover the directional properties of a SMD equipped with two $\pi / 4$ readouts, it is convenient to consider a composite detector made of two co-located TCs with parallel cylindrical axes and rotated in the $\left\{x_{1}, x_{2}\right\}$ plane by $\pi / 4$ with respect to each other.

In figure 4, the plots of $\left|H^{+}\right|$and their ratios (horizontal lines) are shown for different travelling directions of a plus-polarized GW. In figure 4(a), the frequency response of the first two quadrupolar modes of the sample SMD is at about $1740 \mathrm{~Hz}$ and $4650 \mathrm{~Hz}$, and the in-between interval approximately sets the detector bandwidth. In figure $4(b)$, five resonant modes of the sample TC appear with favourable coupling to GW. The $\left|H^{+, \times}\right|$curves for different GW directions are the same apart from a scale factor within the detection band. As 


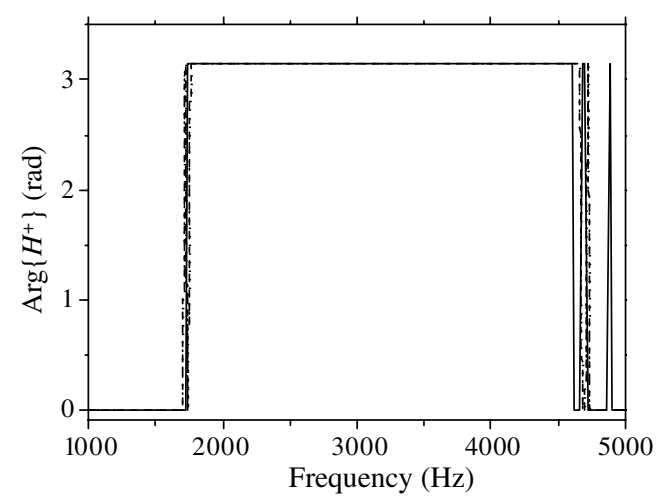

(a)

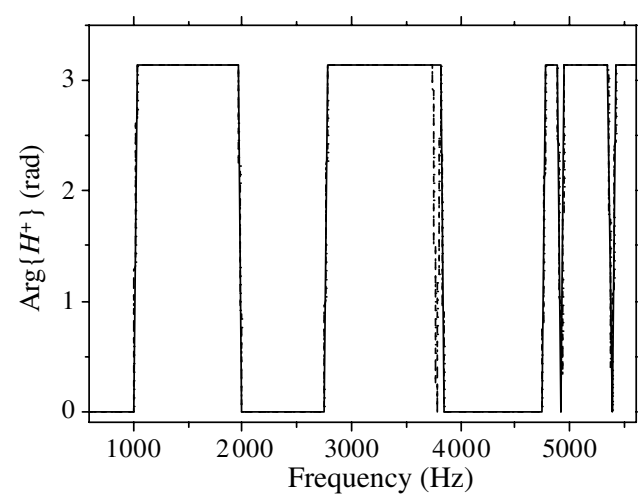

(b)

Figure 5. $\operatorname{Arg}\left\{\mathrm{H}^{+}\right\}$for a plus-wave travelling in the direction: (i) $(\theta=0, \varphi=0)$, solid line; (ii) $\left(\theta=\frac{\pi}{4}, \varphi=0\right)$, dashed line; (iii) $\left(\theta=\frac{\pi}{2}, \varphi=0\right)$, dotted line. The lines described in (ii) and (iii) overlap in almost all the bandwidth. The phase was calculated as $\operatorname{Arg}\left\{H^{+}\right\}=$ $\arccos \left\{\Re\left[H^{+}\right] /\left|H^{+}\right|\right\}$and, as usual, $v=\omega /(2 \pi)$. The lines in the panels $(a)$ and $(b)$ refer to one SMD with a readout as in figure $2\left(a_{1}\right)$ and to one TC, respectively. Similar plots also hold for the cross polarization.

expected, the direction $(\theta=0, \varphi=0)$, i.e. $x_{3}$-axis, represents the optimal direction for the plus- and cross-polarized GWs detected by the readouts implemented on the configurations of figures $2\left(a_{1}\right)$ and $\left(a_{2}\right)$, respectively.

In general, the transfer function of a detector is a complex number. However, for a SMD/TC detector we found that the imaginary part of $H^{+, \times}$is zero in the frequency band between the modes of good GW coupling, within the numerical accuracy of the FEM simulations [34]. Most important, as can be seen in figure 5, is that also the phase of $\mathrm{H}^{+}$ $\left(\operatorname{Arg}\left\{H^{+}\right\}\right)$is independent of the wave direction. To be more precise, $\operatorname{Arg}\left\{H^{+}\right\}$depends on wave travelling directions only in narrow bandwidths around normal modes of the elastic body.

\section{Directional sensitivity and test for monopole rejection}

We discuss here the four antenna patterns $F_{1,2}^{+, \times}$, computed by the FEM simulations, related to plus/cross-polarized waves detected by the first $\xi_{1}^{\text {SMD }}$ and second $\xi_{2}^{\text {SMD }}$ transducer implemented on the configurations of figures $2\left(a_{1}\right)$ and $\left(a_{2}\right)$, respectively. The resulting $F_{1,2}^{+, \times}$are the same if we consider two co-located TC detectors or interferometric detectors rotated by $\pi / 4$.

As expected from the transducer configurations, a plus-polarized $\mathrm{GW}$, impinging on a SMD read by a transducer as in figure $2\left(a_{1}\right)$, produces an antenna pattern represented in figure 6, while the antenna pattern for cross-polarized waves, seen by the same transducer, is shown in figure 7. Both directional responses are equal, within the accuracy of the ANSYS code $(\sim 1 \%)$, to those of an interferometric GW detector, with two orthogonal arms oriented along the $x_{1}$ and $x_{2}$-axes of figures 6 and 7. The transducer shown in figure $2\left(a_{2}\right)$ features the same antenna patterns of figures $6(a)$ and $7(a)$, but rotated by $\varphi=\pi / 4$.

The equivalence between the antenna pattern of a DUAL detector and an interferometer cannot be easily guessed as DUAL is not a resonant detector, and therefore the simple approach of the normal mode expansion is not viable. However, with the help of the FEM simulations, we were able to check that the selective readout allows us the factorization of the complete transfer function in two parts: (1) source position and polarization (antenna pattern), and (2) 


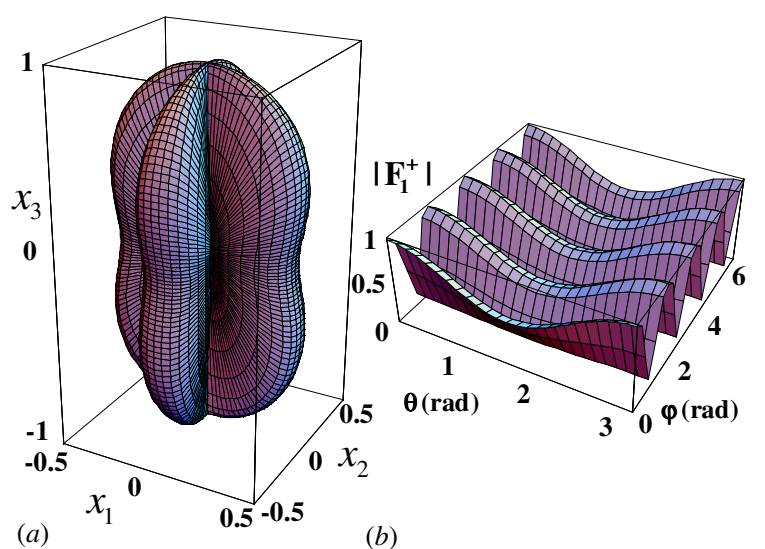

(a)

(b)

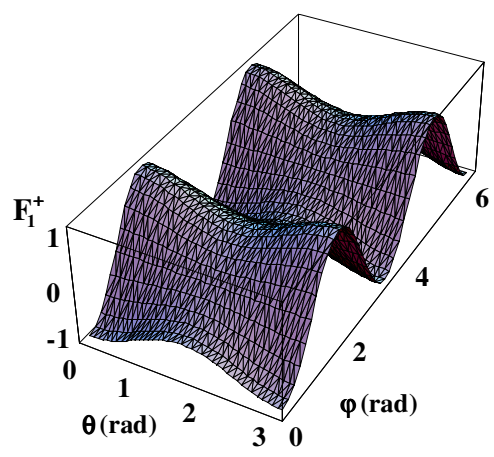

Figure 6. Directional response function, calculated by the FEM simulations, of a SMD equipped with a transducer as in figure $2\left(a_{1}\right)$ or of a TC detector in: $(a)$ Cartesian coordinates $\left\{x_{1}, x_{2}, x_{3}\right\}$ and (b) spherical-polar coordinates $\{r, \theta, \varphi\}$. (c) Real part of the detector response in spherical-polar coordinates. In panel $(a)$, the cylinder is vertically placed at the center of the surrounding box. These plots refer to plus linearly polarized waves. If we define the parametric two-dimensional surface as $r(\theta, \varphi) \equiv\left|F_{1,2}^{+, \times}(\theta, \varphi)\right|$, the distance $r$ from a point of the surface $\{r(\theta, \varphi), \theta, \varphi\}$ to the origin is a measure for the GW sensitivity in the $(\theta, \varphi)$ direction.

GW frequency. By changing the readout system, e.g. by reading only one instead of four sensing surfaces, the resulting antenna pattern is quite complicated and completely different from that of interferometers.

Figure 6 shows that the readout of figure $2\left(a_{1}\right)$ detects plus GWs: (i) with maximum sensitivity when they propagate in the directions $\theta=0$ or $\theta=\pi$ and $\varphi=n \pi / 2$ with $n=1,2,3$, 4; (ii) with half of the maximum sensitivity for $\theta=\pi / 2$ and $\varphi=n \pi / 2$; however, this readout is completely blind to plus GWs coming from $(0<\theta \leqslant \pi, \varphi=(2 n+1) \pi / 4)$ (meridians of the unit sphere).

As shown in figure 7 , the same readout is maximally sensitive to cross waves travelling in the directions $\theta=0$ or $\theta=\pi$ and $\varphi=(2 n+1) \pi / 4$; however, it is blind to cross waves coming from $(0<\theta \leqslant \pi, \varphi=n \pi / 2)$ and $(\theta=\pi / 2,0<\varphi \leqslant 2 \pi)$ (equator of the unit sphere).

The responses of the two readout systems can be combined in different ways, allowing the reconstruction of signal properties when additional information is available. For instance, as in the case of triggered searches, when the wave propagation direction $(\theta, \varphi)$ is known, the SMD with its two independent readout channels, or a pair of TCs, $\pi / 4$ apart, can solve both amplitude polarizations of the incoming GW. As a comparison, let us recall that the proposed 


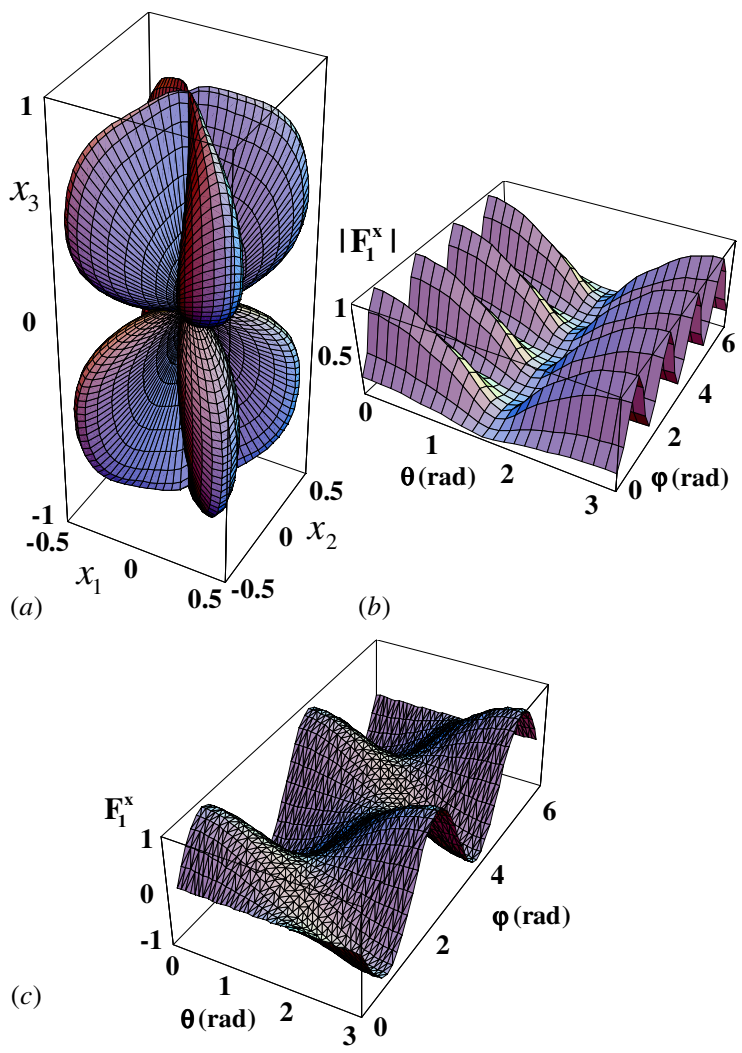

Figure 7. As in figure 6, but for cross-polarized waves.

spherical resonant detectors, equipped with six radial displacement transducers, can measure amplitudes and direction for both GW polarizations, with isotropic sensitivity [35].

\subsection{Circularly polarized GWs}

The antenna patterns for circularly polarized waves detected by the readout systems oriented as in figures $2\left(a_{1}\right)$ and $\left(a_{2}\right)$, given by $F_{1}=\left[\left(F_{1}^{+}\right)^{2}+\left(F_{1}^{\times}\right)^{2}\right]^{1 / 2}$ and $F_{2}=\left[\left(F_{2}^{+}\right)^{2}+\left(F_{2}^{\times}\right)^{2}\right]^{1 / 2}$, are plotted in figures $8(a)$ and $(b)$, respectively. Both responses show that each readout system is completely blind in four perpendicular directions in the equatorial plane, and that the maximum sensitivity is achieved for GWs propagating along the cylinder symmetry axis. For symmetry reasons, $F_{2}$ is equal to $F_{1}$ after a rotation by $\pi / 4$ in the $\left\{x_{1}, x_{2}\right\}$ plane.

The two detector responses can be combined for instance by taking the maximum of the responses of the two readouts (corresponding to independent GW searches in the two readout channels), and so the resulting directional sensitivity can be represented by the envelope of the two antenna patterns of figure 8 (see $[19,34]$ ). Such a synthesis of the two antenna patterns does not show blind directions; thus a SMD detector with two readouts can be considered omnidirectional, even if it is not isotropic: the antenna pattern for GWs coming in the $\left\{x_{1}, x_{2}\right\}$ plane ranges from $30 \%$ to $55 \%$ of the response to an optimally oriented GW. Of course, the same result can be obtained by considering two co-located GW interferometers, rotated by $\pi / 4$. 

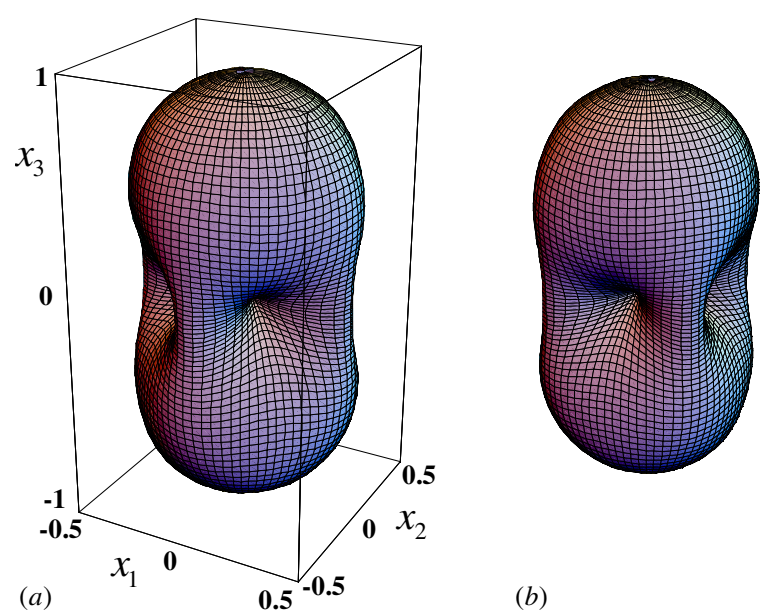

Figure 8. FEM antenna patterns for circularly polarized GWs detected by a transducer as in figures $2\left(a_{1}\right)$ (left) and $\left(a_{2}\right)$ (right).

An alternative synthesis of the readout channels is to consider the quadratic sum of the two responses for circularly polarized waves, i.e. $\left(F_{1}^{2}+F_{2}^{2}\right)^{1 / 2}$. This synthesis shows a performance similar to the previous case, except for the directions in the equatorial plane $(\theta=\pi / 2,0<\varphi \leqslant \pi)$; in fact, the response to GWs coming from any direction in the equatorial plane is $\sim 35 \%$ of the response to an optimally oriented GW.

\subsection{Rejection of monopole disturbances}

To figure out the effectiveness of the selective readout system in rejecting deformations of different geometrical symmetries, we ran a different FEM simulation, i.e. with a trace contribution to the tidal matrix $\boldsymbol{R}$. In particular, we considered the matrix $\hat{\boldsymbol{R}}=$ $\left(\omega^{2} / c^{2}\right) \operatorname{diag}[1,1,1]$ as a model of possible class of spurious external forces. This is the simplest choice for a force perturbation with a monopole contribution, i.e. an isotropic force per unit of mass $\boldsymbol{f}_{\text {iso }}=-\omega^{2} \boldsymbol{x}$. For symmetry reasons, we expect a null response for the assumed selective readout systems $[19,20]$. In fact, the vector field $f_{\text {iso }}$ contains no quadrupolar component. The results of the FEM simulations confirm this expectation, turning out to be zero within the numerical accuracy of the FEM analysis: the selective readout system rejects disturbances which do not have quadrupolar symmetry, even if the spatial projection of quadrupolar normal modes is only approximate.

We note that scalar GWs predicted by scalar-tensor theories of gravity [36] are not rejected by the SMD/TC quadrupolar readout system. In fact, the polarization tensor of a scalar GW, propagating in the $x_{3}$-direction in the transverse gauge, reads $e^{s}=\operatorname{diag}[1,1,0]$ [37] and the tidal matrix representing a scalar GW is $\boldsymbol{R}^{s}=-\ddot{h}_{s}(t) \boldsymbol{e}^{s} /\left(2 c^{2}\right)$, where $h_{s}$ is the amplitude of the scalar transverse wave. Needless to say that scalar or tensor GWs can be discriminated in a detector network.

\section{Solution to the GW inverse problem}

In the previous sections we assigned the $\mathrm{GW}$ and evaluated the directional response of a $\mathrm{SMD} / \mathrm{TC}$ detector equipped with suitable displacement transducers. Now we consider the inverse problem for the detection of GW bursts by using some TCs. In general, a network 
of $N \geqslant 3$ non-aligned detectors provides redundant measurements of the two polarization components of a GW. Thus, there are linear combinations of the detector outputs in which gravitational signals cancel out (null streams), while detector noises and spurious signals remain [14]. Null streams can be used to assess a GW signal without any assumption on its waveform and to distinguish it from a spurious signal. This is the key feature of coherent analysis methods with respect to the time coincidence analyses.

Let us consider $N \mathrm{GW}$ detectors, labelled by $l=1,2, \ldots, N$, at different locations on the Earth identified by the vectors $\boldsymbol{r}_{l}$ joining the Earth center to the $l$ th detector site. We can express the response of the $l$ th detector to an incoming GW, with polarization amplitudes $h_{+, \times}$, as

$$
\xi_{l}(\omega)=\mathrm{e}^{-\mathrm{i} \omega \boldsymbol{k} \cdot \boldsymbol{r}_{l}}\left[F_{l}^{+}(\theta, \varphi) h_{+}(\omega)+F_{l}^{\times}(\theta, \varphi) h_{\times}(\omega)\right]+\eta_{l}(\omega),
$$

where $k$ represents the wave travelling direction, $\eta_{l}$ represents the detector noise that we model as zero mean and independent Gaussian stochastic processes, with variance $\left\langle\eta_{l}(\omega) \eta_{l^{\prime}}\left(\omega^{\prime}\right)\right\rangle=\delta_{l l^{\prime}} S_{h h ; l}(\omega) \delta\left(\omega-\omega^{\prime}\right)$, where $\delta_{l l^{\prime}}$ and $\delta\left(\omega-\omega^{\prime}\right)$ are Kronecker delta and Dirac delta, respectively; $S_{h h ; l}(\omega)$ represents the power spectral density expressed in terms of GW amplitude at the input of the $l$ th detector.

After multiplying each detector response $\xi_{l}(\omega)$ by the phase factor $\mathrm{e}^{\mathrm{i} \omega \boldsymbol{k} \cdot \boldsymbol{r}_{l}}$, which corresponds to the synchronization of all responses on the same wavefront, we can recast equation (4) in the matrix form

$$
\boldsymbol{\xi}(\omega)=\boldsymbol{F}(\theta, \varphi) \cdot \boldsymbol{h}(\omega)+\boldsymbol{\eta}(\omega),
$$

where

$$
\boldsymbol{\xi}(\omega)=\left[\begin{array}{c}
\xi_{1}(\omega) \\
\xi_{2}(\omega) \\
\vdots \\
\xi_{N}(\omega)
\end{array}\right], \quad \boldsymbol{h}(\omega)=\left[\begin{array}{c}
h_{+}(\omega) \\
h_{\times}(\omega)
\end{array}\right], \quad \boldsymbol{\eta}(\omega)=\left[\begin{array}{c}
\eta_{1}(\omega) \\
\eta_{2}(\omega) \\
\vdots \\
\eta_{N}(\omega)
\end{array}\right],
$$

and the network response matrix is given by

$$
\boldsymbol{F}(\theta, \varphi)=\left[\begin{array}{cc}
F_{1}^{+} & F_{1}^{\times} \\
F_{2}^{+} & F_{2}^{\times} \\
\vdots & \vdots \\
F_{N}^{+} & F_{N}^{\times}
\end{array}\right] \equiv\left[\begin{array}{ll}
\boldsymbol{F}^{+} & \boldsymbol{F}^{\times}
\end{array}\right]
$$

When the number of equations is larger than the number of unknown wave parameters, we can use the additional detector outputs as linear constraints, with the aim of discriminating local spurious disturbances from true GWs. To better elaborate this point, let us consider the functional

$$
\Lambda(\boldsymbol{h})=\|\boldsymbol{\xi}(\omega)-\boldsymbol{F} \cdot \boldsymbol{h}(\omega)\|^{2},
$$

where $\|\cdot\|$ is the usual norm of a vector. The minimum of $\Lambda(\boldsymbol{h})$ can be found by means of the Moore-Penrose inverse of the rectangular $N \times 2$ matrix $\boldsymbol{F}$ [38]. The calculation leads to the estimate of the two independent GW polarization components, $h_{+}$and $h_{\times}$[13]. In fact, by differentiating equations (8) with respect to $h$, and setting them equal to zero, we obtain a system of linear equations that, written in the matrix form, reads

$$
\boldsymbol{M} \cdot \boldsymbol{h}(\omega)=\boldsymbol{F}^{T} \cdot \boldsymbol{\xi}(\omega),
$$

where $\boldsymbol{M}=\boldsymbol{F}^{T} \cdot \boldsymbol{F}$. Therefore, the solution to the first part of the inverse problem, i.e. the reconstruction of $h_{+}$and $h_{\times}$, is given by

$$
\boldsymbol{h}(\omega)=\boldsymbol{F}^{\dagger} \cdot \boldsymbol{\xi}(\omega),
$$


where $\boldsymbol{F}^{\dagger} \equiv \boldsymbol{M}^{-1} \cdot \boldsymbol{F}^{T}$ is the Moore-Penrose inverse, or pseudo-inverse, of $\boldsymbol{F}$. The MoorePenrose inverse also provides a least squares solution to the system of linear equations (5). In fact, if detector noises can be described as independent, quasi-stationary Gaussian stochastic processes, one can demonstrate that the minimum of the functional $\Lambda(\boldsymbol{h})$ in equation (8) corresponds to the global maximum of the network likelihood function, defined as the product of the likelihood of each detector in the network [13].

In general, the problem described above is an ill-posed problem. In fact, due to detector alignments in the network, rows of $\boldsymbol{F}$ could be proportional to each others for some source positions in the sky, leading to rank deficiency in $\boldsymbol{F}$. For such positions, $\boldsymbol{h}$ cannot be straightforwardly recovered by means of $\boldsymbol{F}^{\dagger}$. The condition number of $\boldsymbol{F}$, expressed by $\operatorname{cond}(\boldsymbol{F}) \equiv\|\boldsymbol{F}\|\left\|\boldsymbol{F}^{\dagger}\right\|{ }^{6}$ gives an estimate of how the matrix is close to these singularities. An ill-conditioned matrix, i.e. with $\operatorname{cond}(\boldsymbol{F}) \gg 1$, produces a high instability in the solution of the inverse problem. In particular, the condition number associated with the linear equation (5) gives a bound on how inaccurate the solution $\boldsymbol{h}$ will be due to noise $\boldsymbol{\eta}$. We can roughly consider the condition number as the rate at which the solution $\boldsymbol{h}$ will change with respect to a change in the responses $\boldsymbol{\xi}$. Thus, if the condition number is large, even a small error in $\boldsymbol{\xi}$ (high signal-to-noise ratio) may cause a large error in $\boldsymbol{h}$. On the other hand, if the condition number is small then the error in $\boldsymbol{h}$ will not be much larger than the noise fluctuations in $\boldsymbol{\xi}$. It is worth noting that the conditioning is a property of the matrix $\boldsymbol{F}$, not of the algorithm used to reconstruct the source position and polarization.

Many methods have been devised to face ill-posed problems; among them the most noticeable is the regularization method proposed by Tikhonov [40].

The solution of the inverse problem not only reconstructs the GW polarization amplitudes $h_{+, \times}$and its direction $k(\theta, \varphi)$ but it can also discriminate genuine from spurious signals by means of two suitable statistics. In fact, we can introduce two linear operators, $\boldsymbol{P}=\boldsymbol{F} \cdot \boldsymbol{F}^{\dagger}$ and $\boldsymbol{Q}=\boldsymbol{I}-\boldsymbol{F} \cdot \boldsymbol{F}^{\dagger}$, where $\boldsymbol{I}$ is the $N \times N$ identity matrix. One can easily show that $\boldsymbol{P}$ and $\boldsymbol{Q}$ are two projection operators, orthogonal and complementary. The operators $\boldsymbol{P}$ and $\boldsymbol{Q}$ project the detector outputs in the vectorial space spanned by $\boldsymbol{F}$ and in the null space of $\boldsymbol{F}$, i.e. the space orthogonal to $\boldsymbol{F}$, respectively. Then, for each sky position, we can define two positive definite statistics: the network power $E(\theta, \varphi) \equiv\|\boldsymbol{P} \cdot \boldsymbol{\xi}\|^{2}$ and the residual function $R(\theta, \varphi) \equiv\|\boldsymbol{Q} \cdot \boldsymbol{\xi}\|^{2}$. The corresponding expected values, under the hypothesis of a true GW, reach a maximum and a minimum, respectively [14]; therefore the desired source location and spurious discrimination can be obtained by requiring the simultaneous level crossing of $E(\theta, \varphi)$ and uncrossing of $R(\theta, \varphi)$.

\section{TCs and LIGO-VIRGO-TC networks}

\subsection{Homogeneous network}

In the case of $N=2$ aligned TC detectors, located in the same place, the vectors $\boldsymbol{F}^{+}$ and $\boldsymbol{F}^{\times}$represent the whole two-dimensional space, therefore the complementary space, i.e. the null space, consists of the null vector. The solution of the problem is represented by equations (10), with $\boldsymbol{F}^{\dagger}=\boldsymbol{F}^{-1}$, because in this case $\boldsymbol{F}$ is a square matrix of rank 2 . Therefore, $\boldsymbol{P}=\boldsymbol{F} \cdot \boldsymbol{F}^{-1}, \boldsymbol{Q}=0$ and $R(\theta, \varphi)=0$ for all $(\theta, \varphi)$, then it is not possible either to infer the source position or to discriminate spurious events. It would need at least another detector to solve the problem. In fact, in the case of $N=4 \mathrm{TC}$ detectors, the vectors $\boldsymbol{F}^{+}$and $\boldsymbol{F}^{\times}$ generate a two-dimension subspace, and then a two-dimensional complementary subspace (i.e. the null space) exists [14]. If we arrange the four TCs in two pairs to form two equivalent

6 In calculating cond $(\boldsymbol{F})$ we used the induced matrix 2-norm (see e.g. [39]). 


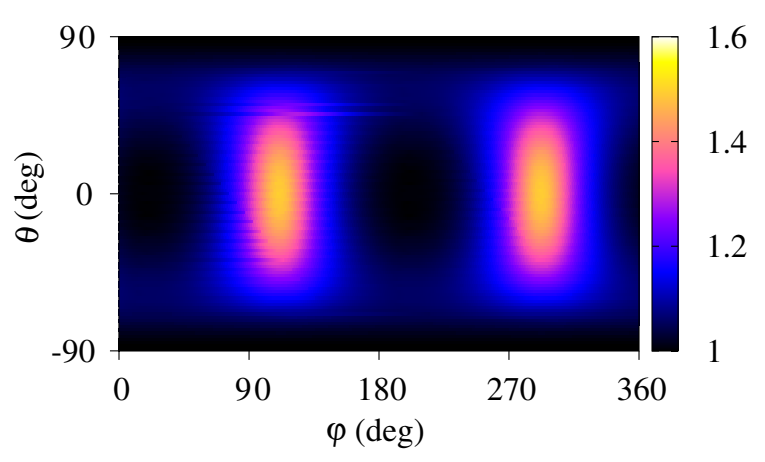

Figure 9. Sky map of the condition number for a homogeneous network of $N=4$ TCs. The four detectors form two pairs with symmetry axes aligned along the $(\theta=\pi / 2, \varphi=0)$ and $(\theta=\pi / 2, \varphi=\pi / 4)$ directions.

SMD detectors, and we align their symmetry axes to form a $\pi / 4$ angle, we have the very good conditioning shown in figure 9. Another notable feature of such a homogeneous network is the invariance of the condition number for rigid rotation of each SMD about its symmetry axis.

In any case, if the polarization of gravitational signals were known, it would be possible to estimate the wave direction by means of a pair of TC detectors. In fact, in this case, the null space would be one dimensional and so we could form the $E(\theta, \varphi)$ and $R(\theta, \varphi)$ statistics.

As we have already said, a TC is equivalent in terms of antenna pattern to an interferometer with two orthogonal arms. Thus, instead of considering a homogeneous network made of several TCs, we think that it is more plausible to investigate the integration of one highly sensitive TC in the advanced LIGO-VIRGO network, as discussed in the next paragraph.

\subsection{Heterogeneous network}

A network of advanced interferometric detectors will be in operation by the next halfdecade. The bandwidth of these high-sensitivity detectors will reach $\sim 5 \mathrm{kHz}$ and will overlap significantly with that of a TC with comparable sensitivity $\left(\gtrsim 10^{-23} \mathrm{~Hz}^{-1 / 2}\right)$ in the range $\sim(2-5) \mathrm{kHz}$. Then, it is worth investigating the contribution of a TC to the advanced interferometric network.

In figure 10, the power spectral sensitivities expected for a Mo TC, advanced LIGO and VIRGO detectors are shown. The performance of a TC detector in terms of strain sensitivity could be improved by considering a material with a coupling to GWs higher than that of Mo, such as for instance the $\mathrm{C} / \mathrm{SiC}$ material [20, 42].

As a possible site for operating a TC, we considered the INFN-National Laboratory of Gran Sasso (LNGS) in Italy. The remaining degree of freedom of a TC orientation is the azimuth angle $\zeta$ between the local north direction and the readout system, measured clockwise in the horizontal plane. Figure 11 shows that a TC detector improves considerably the condition number of the network and that the best conditioning is achieved at $\zeta \sim 75^{\circ}$. To appreciate the reconstruction ability of the LIGO-VIRGO-TC network, we show in figure 12 the condition number as a function of source angles $\theta$ and $\varphi$, with a TC located at LNGS and $\zeta \sim 75^{\circ}$.

The very low achieved values of $\operatorname{cond}(\boldsymbol{F})$ clearly indicate that the solution of the inverse problem is stable and that $R(\theta, \varphi)$ can be estimated all over the sky. To show the TC contribution to network performances, we report in table 2 the results of a Monte Carlo 


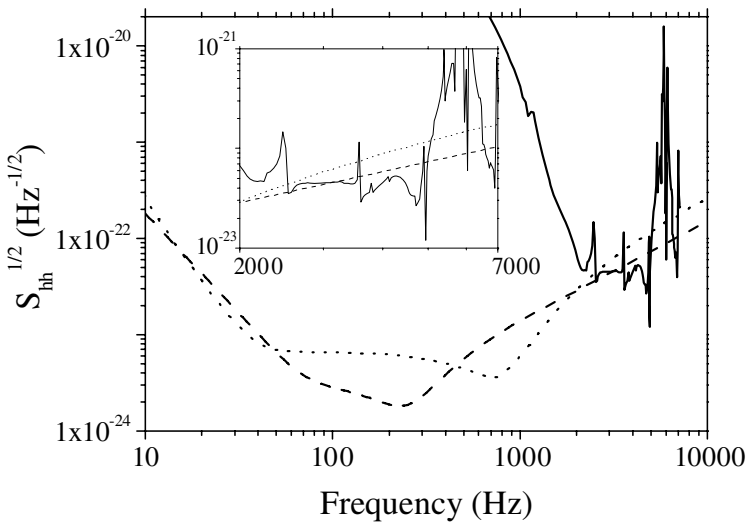

Figure 10. Standard quantum limit strain sensitivities for the Mo TC detector of figure 3 (solid curve), advanced LIGO (dashed curve) [41] and advanced VIRGO (dotted curve). The inset represents an enlargement of the noise spectra in the (2-7) $\mathrm{kHz}$ frequency band; $v=\omega /(2 \pi)$.

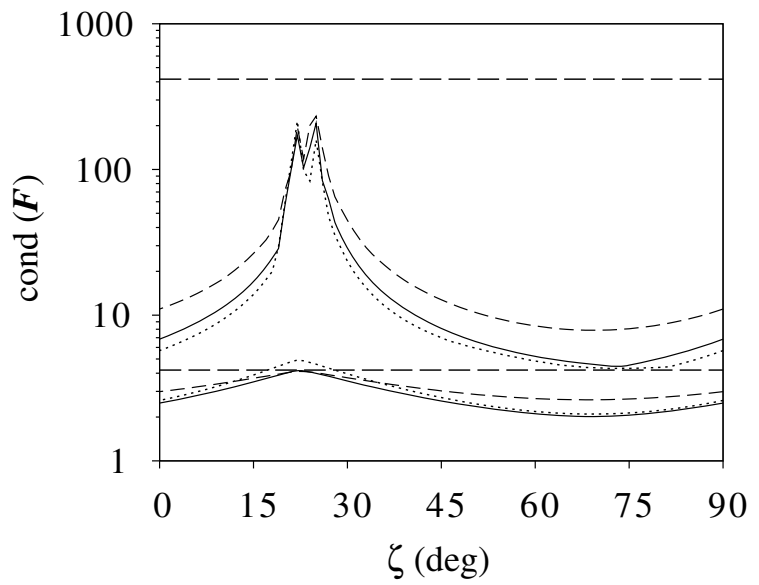

Figure 11. Condition number of the response matrix for the LIGO-VIRGO-TC network, with the TC detector located at LNGS, as a function of the local azimuth angle $\zeta$. The solid, dashed and dotted lines refer to a TC detector with the same, half or double strain sensitivity, respectively. The three upper and lower lines show the worst and the average conditioning, respectively. The two dashed-dotted horizontal lines represent the worst (upper line) and the average (lower line) conditioning of the advanced LIGO-VIRGO network. The average conditioning is evaluated assuming a uniform source distribution.

simulation of the fraction of the sky covered by the two network configurations, where the conditioning is lower than a given threshold. The Monte Carlo consists of $10^{5}$ random directions of the sky, uniformly distributed over the unit sphere. As clearly evident from table 2, a LIGO-VIRGO-TC network better reconstructs GW signals irrespective of their direction and polarization, within the TC frequency band.

It is worth noting that the improvement of adding SMD/TC detectors to the LIGO-VIRGO network comes from the ability to search for both polarizations, thanks to the optimization of the $\zeta$ angle. 


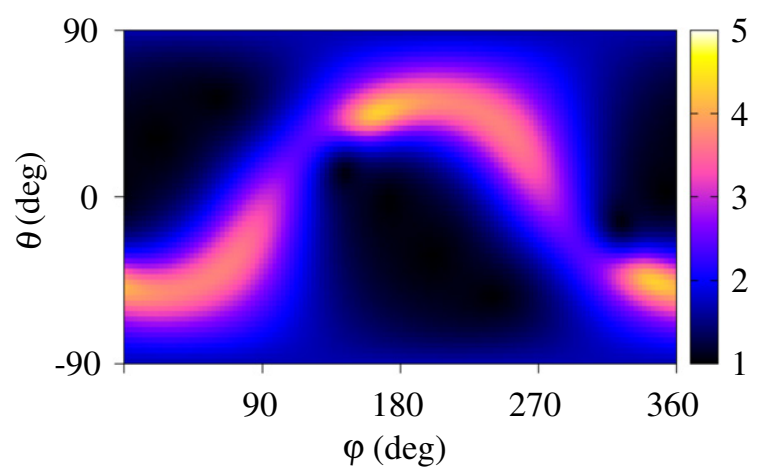

Figure 12. Sky map of the condition number for the LIGO-VIRGO-TC network, assuming the best TC orientation $\zeta \sim 75^{\circ}$.

Table 2. Fraction of the sky where conditioning is less than the given threshold of maximum conditioning (Max Cond). We assume the same sensitivity for the advanced LIGO/VIRGO and TC detectors in the $\sim(2-5) \mathrm{kHz}$ band and the best orientation angle $\zeta \sim 75^{\circ}$ for the TC detector.

\begin{tabular}{llc}
\hline Max Cond & LIGO-VIRGO $(\%)$ & LIGO-VIRGO-TC $(\%)$ \\
\hline 4.5 & 73 & 100 \\
4.0 & 67 & 99 \\
3.5 & 59 & 93 \\
3.0 & 48 & 85 \\
2.5 & 36 & 73 \\
2.0 & 22 & 58 \\
1.5 & 7 & 39 \\
\hline
\end{tabular}

\section{Conclusions}

In this paper we discussed the directional sensitivity to GWs of a single-mass DUAL (SMD) and a tapered cylinder (TC) equipped with selective readout. The dynamics of the detectors was simulated on the basis of three-dimensional FEM numerical analyses, by computing the $\mathrm{SMD} / \mathrm{TC}$ transfer function $H^{+, \times}(\theta, \varphi ; \omega)$ to plus- and cross-polarized GWs coming from any direction. The main result was the factorization of $H^{+, \times}$in the angular and frequency dependences within the $\sim(2-5) \mathrm{kHz}$ detection band. Such a factorization is due to the selective quadrupolar readout configuration and allows one to compute the SMD/TC antenna pattern. We showed that a SMD, or a pair of TC detectors with parallel symmetry axes, but rotated by $\pi / 4$ one with respect to the other, does not have blind directions in the sky. In addition, we analysed an example of rejection of monopole spurious signal by a SMD/TC in confirmation of the capability of the selective readout system to reject disturbances which do not have a quadrupolar symmetry.

We also discussed the inverse problem of detecting and reconstructing gravitational signals by exploiting the FEM frequency-dependent harmonic analyses for a couple of TCs, $\pi / 4$ apart.

In this framework, we investigated the possibility of adding a TC in the network of advanced LIGO-VIRGO detectors, since the expected strain sensitivities of the detectors are comparable above $2 \mathrm{kHz}$. The improvements of detection performances of such a network are of some relevance. In fact, the region of likely detection is significantly extended and the sky 
coverage of the overall network is high enough: the resulting sky map exhibits the worst case corresponding to a condition number of 4.5. The same figure for the conditioning is achieved by the LIGO-VIRGO network on $\sim 70 \%$ of the sky.

\section{Acknowledgment}

One of the authors (PL) acknowledges the support of 'Consorzio Criospazio Ricerche', Trento.

\section{References}

[1] Astone P et al 2007 Phys. Rev. D 76102001

[2] Abbott B et al 2007 Class. Quantum Grav. 245343

[3] Baggio L et al 2008 Class. Quantum Grav. 25095004

[4] Abbott B et al 2008 Phys. Rev. D 77062004

[5] Abbott B et al 2007 Phys. Rev. D 76062003

[6] Baggio L et al 2005 Phys. Rev. Lett. 95081103

[7] Gursel Y and Tinto M 1989 Phys. Rev. D 403884

[8] Gottardi L 2007 Phys. Rev. D 75022002

[9] Cerdonio M, Conti L, Lobo J A, Ortolan A, Taffarello L and Zendri J P 2001 Phys. Rev . Lett. 87031101

[10] Dhurandhar S and Tinto M 1988 Mon. Not. R. Astron. Soc. 234663

[11] Tinto M and Dhurandhar S 1989 Mon. Not. R. Astron. Soc. 236621

[12] Finn L S 2001 Phys. Rev. D 63102001

[13] Klimenko S, Mohanty S, Rakhmanov M and Mitselmakher G 2005 Phys. Rev. D 72122002

[14] Chatterji S, Lazzarini A, Stein A, Sutton P J, Searle A and Tinto M 2006 Phys. Rev. D 74082005

[15] LIGO http://www.ligo.caltech.edu/

[16] Sigg D (for the LSC) 2006 Class. Quantum Grav. 23 S51

[17] VIRGO http://www.virgo.infn.it/

[18] Rakhmanov M 2006 Class. Quantum Grav. 23 S673

[19] Bonaldi M, Cerdonio M, Conti L, Falferi P, Leaci P, Odorizzi S, Prodi G A, Saraceni M, Serra E and Zendri J P 2006 Phys. Rev. D 74022003

[20] Leaci P, Vinante A, Bonaldi M, Falferi P, Pontin A, Prodi G A and Zendri J P 2008 Phys. Rev. D 77062001

[21] Bonaldi M, Cerdonio M, Conti L, Prodi G A, Taffarello L and Zendri J P 2004 Class. Quantum Grav. 21 S1155

[22] Bonaldi M, Cerdonio M, Conti L, Pinard L, Prodi G A, Taffarello L and Zendri J P 2003 Phys. Rev. D 68102004

[23] Misner C W, Thorne K S and Wheeler J A 1973 Gravitation (San Francisco, CA: Freeman)

[24] ANSYS, Inc., Southpointe, 275 Technology Drive, Canonsburg, PA 15317 http://www.ansys.com

[25] Meirovitch L 1970 Methods of Analytical Dynamics (New York: McGraw-Hill)

[26] Saulson P 1994 Fundamentals of Interferometric Gravitational Wave Detectors (Singapore: World Scientific)

[27] Duffy W Jr 1992 J. Appl. Phys. 725628

[28] Leaci P and Ortolan A 2007 Phys. Rev. A 76062101

[29] Rapagnani P 1982 Nuovo Cimento C 5385

[30] Mezzena R, Vinante A, Falferi P, Vitale S, Bonaldi M, Prodi G A, Cerdonio M and Simmonds M B 2001 Rev. Sci. Instrum. 723694

[31] Richard J P 1992 Phys. Rev. D 462309

[32] Briant T, Cerdonio M, Conti L, Heidmann A, Lobo A and Pinard M 2003 Phys. Rev. D 67102005

[33] Marin F, Conti L and De Rosa M 2003 Phys. Lett. A 30915

[34] Leaci P Development of wideband acoustic gravitational wave detectors PhD Thesis

[35] Merkowitz S M and Johnson W W 1997 Phys. Rev. D 567513

[36] Brans C and Dicke R H 1961 Phys. Rev. 124925

[37] Maggiore M and Nicolis A 2000 Phys. Rev. D 62024004

[38] Hansen P C 1998 Rank-Deficient and Discrete Ill-Posed Problems: Numerical Aspects of Linear Inversion (Philadelphia, PA: SIAM)

[39] Horn R A and Johnson C R 1985 Matrix Analysis (Cambridge: Cambridge University Press)

[40] Strang G 1998 Introduction to Linear Algebra 3rd edn (Cambridge: Wellesley-Cambridge Press)

[41] http://www.astro.cf.ac.uk/geo/advligo/

[42] Cesic by ECM http://www.cesic.de 